\title{
AHP FOR EVERYBODY: INNOVATION THROUGH MOBILE APPLICATION FOR PERSONAL DECISIONS
}

\begin{abstract}
The paper describes an Analytic Hierarchy Process (AHP) based mobile application for day to day use. Growing internet enabled information reach to individuals via smartphone has created potential for individual decision support systems. Automation and productivity enhancement to people is converging in smartphones. There exists software to process multiple criteria decisions for enterprise level application. However, it is observed that there is not yet a simple user friendly, theory-based decision support application with visualization for individuals. The product makes a theory based scientific multi criteria decision support tool available for common people to easily use by automating the process through an interactive interface. Up until now, the experience of such software was either geared towards an enterprise, or someone who understood the theoretical backing. The mobile application is perceived to be the first of its kind with features of dynamic sensitivity analysis. Change in ranking of alternatives can be instantly seen while changing weight of criteria, the feature is called decision visualization. Considering continuous improvement with the user feedback, a mobile application called DecisionMentor is released and available in application stores for common people's use.
\end{abstract}

Keywords: personal decision support, mobile application, DecisionMentor, commoner, decision visualization

\section{Introduction}

Individuals make on an average 35,000 decisions each day (Jagoda et al. 2020), out of that not all the choices are important and critical. However, decisions like choosing a job, deciding a fiancé, property decisions, vacation decisions, relocation / migration decisions, decision related to job, bank's service reliability, company to shift, airlines to travel, whose service to take, better route to travel, stock selection, wedding location decisions etc. are critical personal decisions. The personal and real-life decisions are mostly multiple criteria decision problems in nature.

Smartphones with multiple functions converged into a single device. A typical cell phone is used in game playing; music, video, radio, watching or listening; camera for photo / video capturing and processing; date, time and scheduling, personal finance management; internet browsing; reading; email processing, communication, social media applications, video meeting etc. are commonly available and used. There are numerous specific purpose applications available in the stores to use with smartphones. One can find huge number of applications while searching; however, we could not find commonly usable theory-based decision support application with visualization feature. The decision support app is perceived useful when one is feeling what to do, and also when there is no one to ask or take advice for decisions or the decision is so private that one could not ask 
ISAHPArticle: A Style Guide for Paper Proposals To Be Submitted to the International Symposium on the Analytic Hierarchy Process 2020, Web Conference.

anyone. The concept of DecisionMentor application is just to bridge the gap to support decisions for individuals in a very user friendly manner.

The paper is all about the making of an AHP based mobile application called DecisionMentor to fulfill the gap identified. Review of available AHP based mobile applications for individuals is featured. Aspects of continuous improvement application are also mentioned.

\section{AHP based Mobile Application Review}

Rapid review at the mobile application stores for theory-based decision support mobile applications conducted with key features like theory used, user friendliness, decision visualization etc. to identify the gap. It is so far noticed that none have dynamic sensitivity analysis for decision visualization. Details on the survey will be presented in full paper.

\section{Objectives}

Our work is to bring something innovative based on AHP for common people. The specific objective is to take AHP to every one's pocket. Thomas Saaty always wanted AHP for solving common people's problems. Taking AHP to general people is possible only when we are able to make an application where the interactions are so easy to use, that the person using it doesn't even have to know the intricacies of AHP.

\section{Methodology of Application Development}

DecisionMentor development process becomes long, if it is counted from ideation; it is about ten year old. The timing to launch became appropriate just after the opening from the Covid-19 lockdown. The intense discussion on the application development started just a few years back between the co-creators. Covid-19 lockdown period intensified development process with trial and error for four months. We rushed to release, thinking that there will never be a final product, continuous improvement is the part of any technology product and services. The trial and error process of development methodology will be discussed in the full paper.

DecisionMentor uses theoretical backing from AHP in its backend, with a simple interaction for the user to help people with phones to make calculated decisions easily. Smartphones have become ubiquitous to daily life in the past decade where people turn to its use at many different touch points during the day. Some simple decisions, like what to buy on an ecommerce site, or what to watch on Netflix burdens a lot of people with the amount of choices they have. This presents us with the perfect timing and opportunity to introduce solutions in the daily life that uses a robust theoretical backing, to help ease people's decision. The best part about using AHP is that, it doesn't alienate the user's criteria's but puts weight to them making them feel like the decision is coming from them and not from a machine. Thus using this to opportunity to advantage, the application is to 
ISAHPArticle: A Style Guide for Paper Proposals To Be Submitted to the International Symposium on the Analytic Hierarchy Process 2020, Web Conference.

be developed with a regular person's day to day life in current days, and the points in their day when they need to make decisions where the application can help.

\section{Result and Discussions}

The mobile application is developed using the Flutter framework, a cross platform application development framework by Google which uses Dart as a programming language. Flutter enables developing mobile applications for Android and iOS using a single code base.

The application building process is iterative, and follows experience design frameworks where target audience and touch points for their day to day use are identified. A functional version of the app with useable interface is release, and based on the feedback, the target-audience and the identified touch points modifications are made iteratively and tested, while using the momentum gained while testing to improve and introduce the application to the world.

Screenshot of DecisionMentor (https://www.decisionmentor.app/), the AHP based Mobile Application for Individual / Personal Decision Support is presented following page.

DecisionMentor's initial version can take only five criteria and five alternatives. The initial version comes with a single level hierarchy. The current version does not display consistency ratio. The current version is limited in both feature and resources, to test and deploy its solution. The joint venture of the development team is still exploring the sustainable business model for the product and services.

\section{Conclusions}


Developing a smart AHP based mobile application and making it available at the mobile app stores is considered a milestone. It is taken as an innovative approach to take AHP to the commoner. The application needs continuous improvement. Continuous improvement of the application is for user experience enhancement and extension of functionalities. Making the application reach a wider population using lingua franca are key aspects of the improvement. Terms used in the application needing to avoid scientific or technical jargons. The work is an important contribution to bring AHP in everyday practice as a productivity tool for individuals.

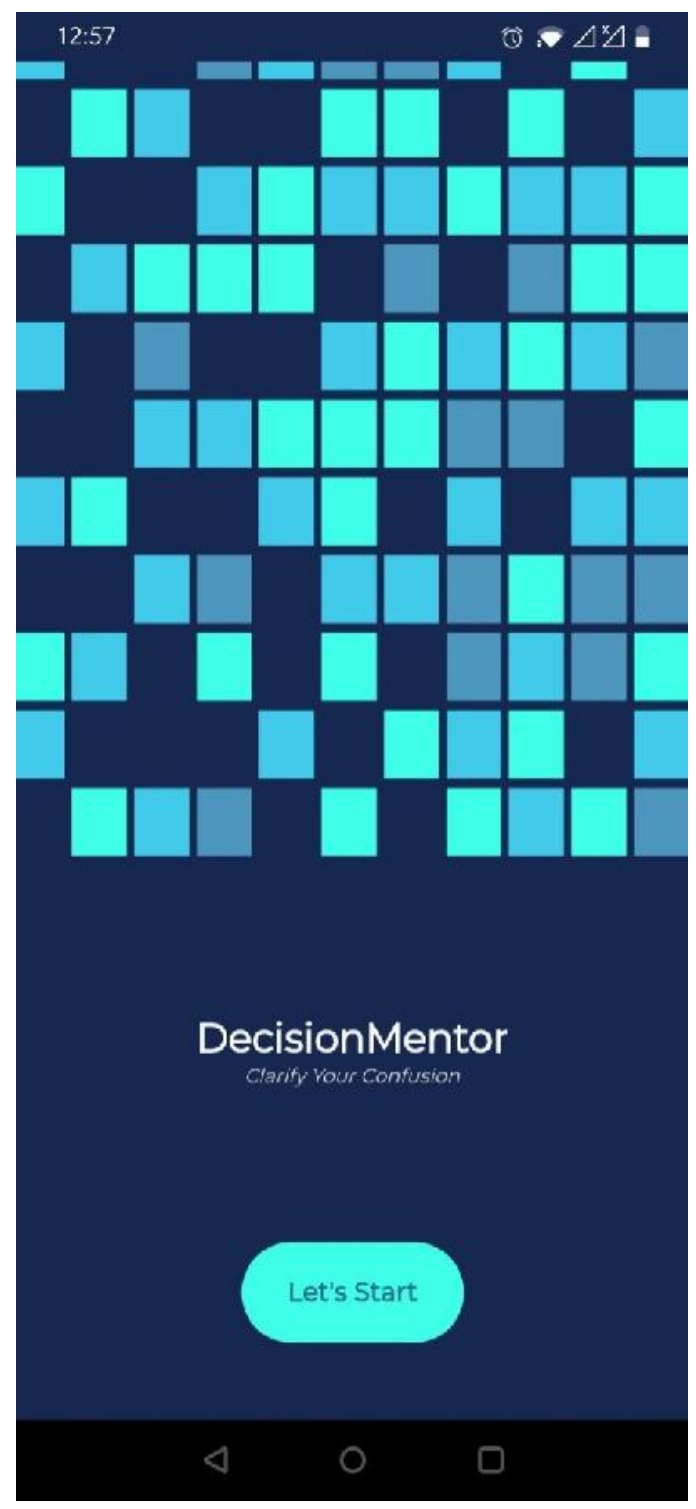

International Symposium on the Analytic Hierarchy Process

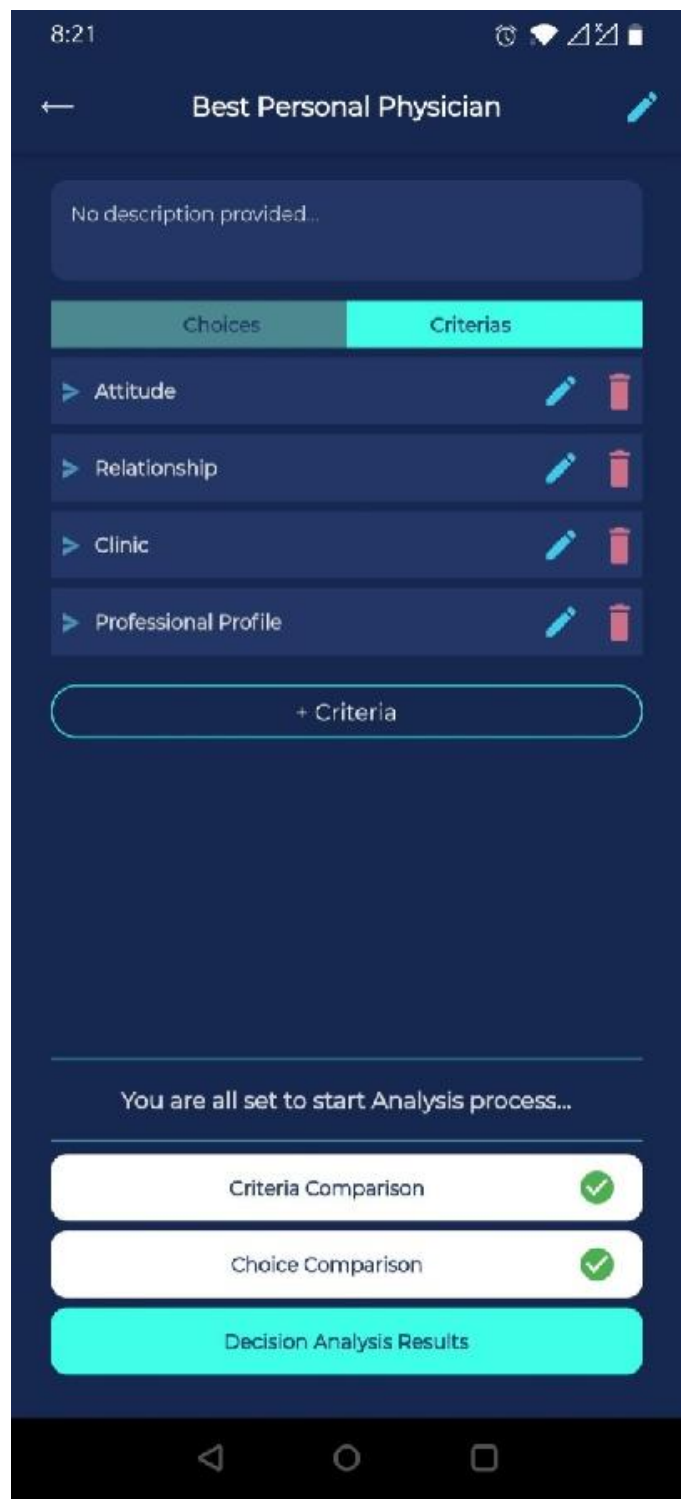


ISAHPArticle: A Style Guide for Paper Proposals To Be Submitted to the International Symposium on the Analytic Hierarchy Process 2020, Web Conference.
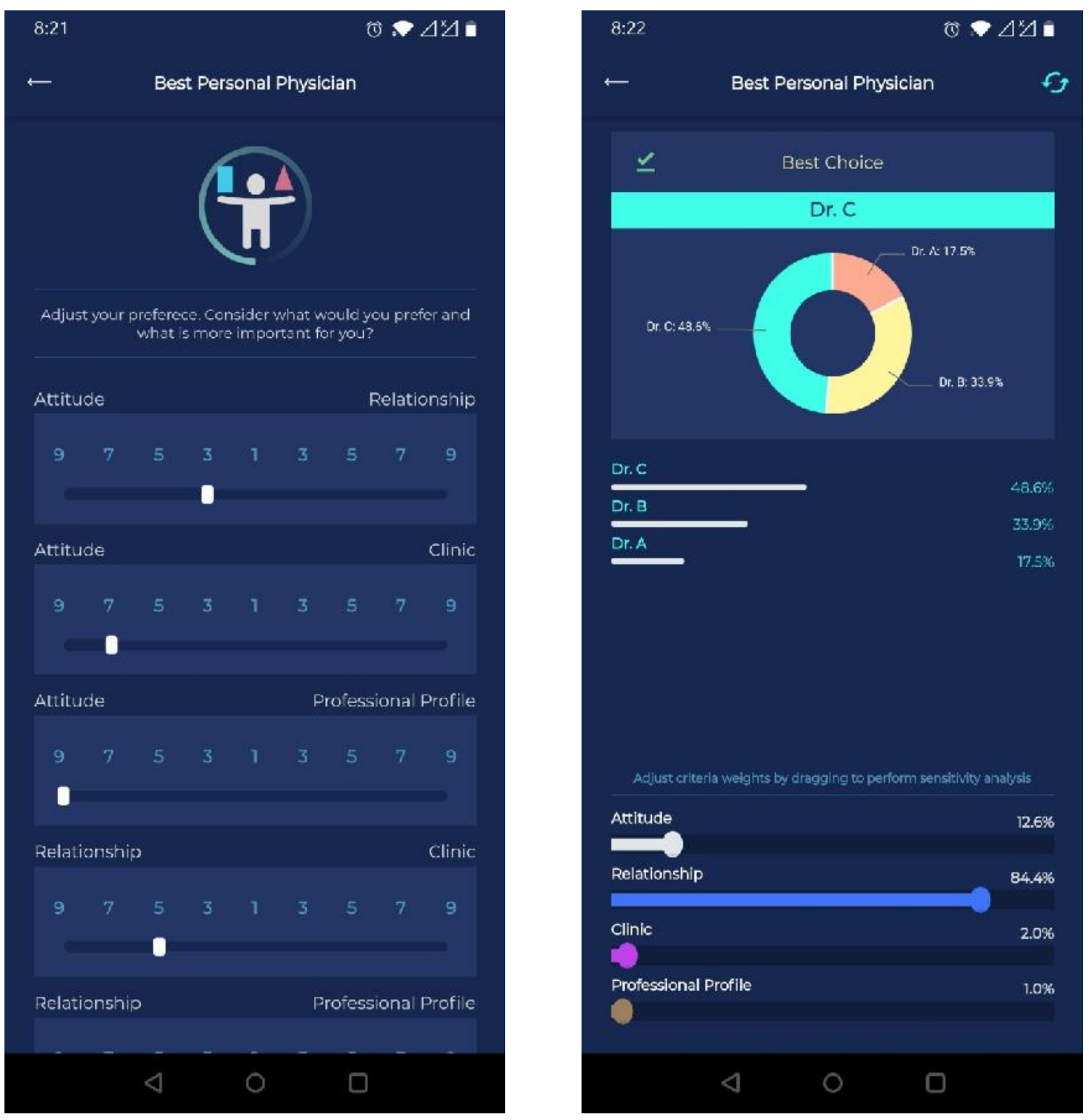

\section{References}

Jagoda J, Schuldt S and Hoisington A (2020). What to Do? Let's Think It Through! Using the Analytic Hierarchy Process to Make Decisions. Front. Young Minds. 8:78. doi: 10.3389/frym.2020.00078) 